
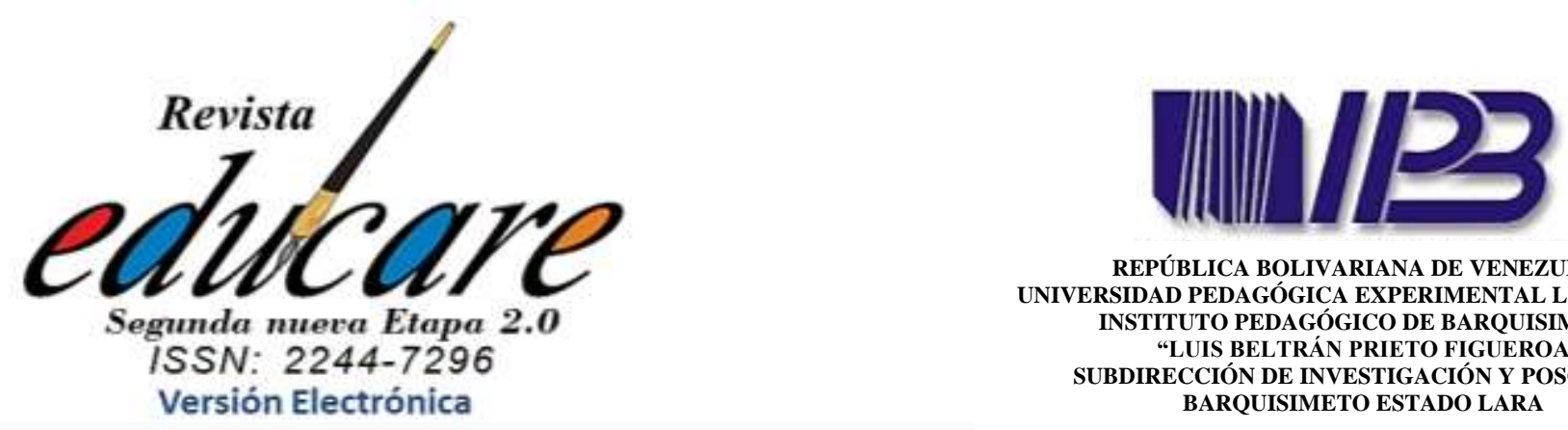

REPÚBLICA BOLIVARIANA DE VENEZUELA UNIVERSIDAD PEDAGÓGICA EXPERIMENTAL LIBERTADOR INSTITUTO PEDAGOGICO DE BARQUISIMETO "LUIS BELTRÁN PRIETO FIGUEROA

SUBDIRECCIÓN DE INVESTIGACIÓN Y POSGRADO BARQUISIMETO ESTADO LARA

Volumen 24 № 1 Enero-Abril 2020

$(270-280)$

Ximena Patricia León Quinapallo

ORCID: https://orcid.org/0000-0002-0258-1350

Norma Amabilia Ortiz Bravo ORCID: https://orcid.org/0000-0002-8324-1699

Richard Manuel Manangón Pesantez

ORCID: https://orcid.org/0000-0002-6739-883X

UNIVERSIDAD CENTRAL DEL ECUADOR ECUADOR

*Docente Universidad Central del Ecuador xpleon@uce.edu.ec

**Docente Universidad Central del Ecuador naortiz@uce.edu.ec

***Docente Universidad Central del Ecuador rmmanangon@uce.edu.ec

\section{CURRÍCULO DE LOS NIVELES DE EDUCACIÓN OBLIGATORIA: UNA MIRADA REFLEXIVA DESDE EL HACER DOCENTE}

\author{
COMPULSORY EDUCATION LEVELS \\ CURRICULUM: A THOUGHTFUL LOOK FROM \\ TEACHING
}

Recibido:

27-11-2019

Aceptado:

$16-01-2020$ 

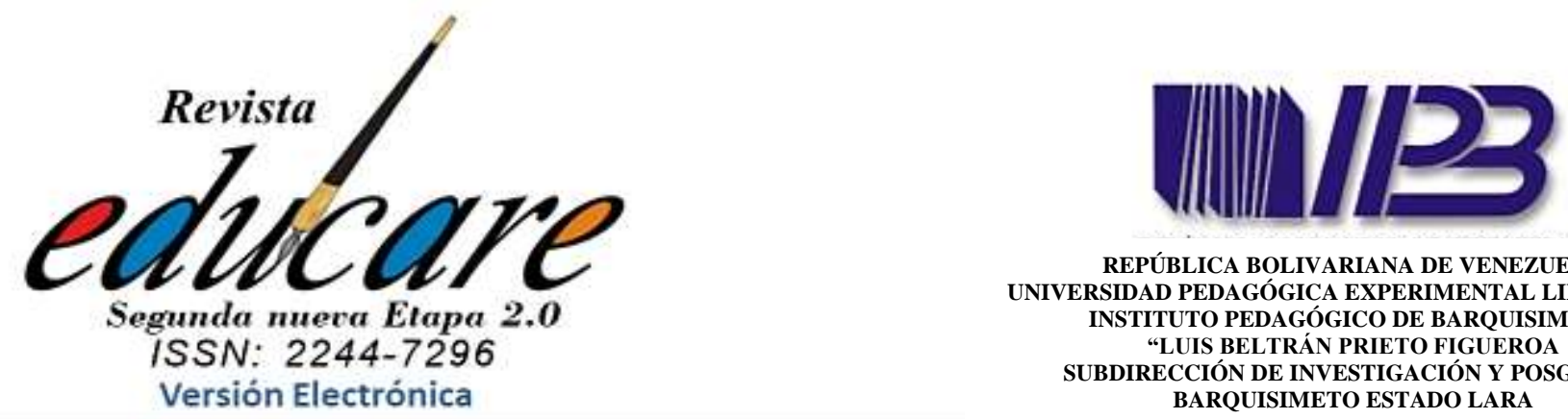

REPÚBLICA BOLIVARIANA DE VENEZUELA UNIVERSIDAD PEDAGÓGICA EXPERIMENTAL LIBERTADOR INSTITUTO PEDAGÓGICO DE BARQUISIMETO "LUIS BELTRÁN PRIETO FIGUEROA

SUBDIRECCIÓN DE INVESTIGACIÓN Y POSGRADO BARQUISIMETO ESTADO LARA

\section{Resume}

El aporte de este artículo se centra en una postura crítica y reflexiva sobre currículo de los niveles de educación obligatoria, específicamente el de Educación Física, el cual es presentado por Ministerio de Educación del Ecuador, la modalidad de esta publicación es de tipo ensayo, y en este se define el currículo como una propuesta innovadora, abierta y flexible, en que el futuro bachiller estará en condiciones de ser autónomo de la práctica de la actividad física, planteado desde el perfil del bachiller ecuatoriano, en base a la necesidad de la sociedad, diseñando tres perfiles en las cuales los futuros ciudadanos tengan la competencia en Justicia, solidaridad e innovación. Después de haber hecho una revisión profunda al currículo, nos permite establecer una posición crítica que posterior nos lleva a la reflexión, dado a que los principios filosóficos, pedagógicos, sociales, etnográficos, psicológicos del documento base no están enmarcadas al planteamiento de cada subnivel. También hay que señalar que las destrezas con criterio de desempeño no están bien construidas, siguiendo una secuencia lógica, y por lo tanto no siempre reflejan un orden progresivo además de tener una contextualización alejada de la realidad del país.

Descriptores: Currículo; Educación Física

\section{Abstract}

The contribution of this article focuses on a critical and reflective stance on curriculum of mandatory levels of education, specifically that of Physical Education, which is presented by the Ministry of Education of Ecuador, the modality of this publication is essay type, and in this the curriculum is defined as an innovative, open and flexible proposal, in which the future bachelor will be able to be autonomous from the practice of physical activity, raised from the profile of the Ecuadorian baccalaureate, based on the need of society, designing three profiles in which future citizens have competence in Justice, Solidarity and Innovation. Having made a thorough review of the curriculum, it allows us to establish a critical position that leads us to reflection, since the philosophical, pedagogical, social, ethnographic, psychological principles of the base document are not framed to the approach of each sublevel. It should also be noted that performance skills are not well constructed, following a logical sequence, and therefore do not always reflect a progressive order in addition to having a contextualization away from the reality of the country.

Keywords: Curriculum; Physical Education 


\section{ESCENARIO DE INTERÉS}

El Currículo Nacional de Educación Física se define como una propuesta innovadora, abierta y flexible, en que el futuro bachiller estará en condiciones de ser autónomo de la práctica de la actividad física, por lo que tuvo que pasar un proceso de la aplicación de la contextualización de los contenidos curriculares con el propósito de adoptarlo a los aprendizaje de los estudiantes, ; en este sentido se le visualiza como un currículo diferente e inentendible para muchos docentes, debido a que los anteriores currículos eran rígidos y cerrados, donde los docente solo se remitan a trabajar en clase los contenidos curriculares temporalizados y preestablecidos, es decir si en la semana de fútbol se debía trabajar pase del balón con borde interno y externo,

De allí que, en todas las instituciones del país se debía trabajar esa técnica deportiva, sin importar que en la institución educativa exista o no un espacio con las condiciones para practicarlo, sin importar que exista o no material para trabajarlo, sin importar las necesidades e intereses de los estudiantes, sin importar si el docente conoce o no estos contenidos, sin importar que exista o no docentes con carga horaria de Educación Física, sin importar que la institución educativa sea unidocente, bidocente, pluridocente, intercultural bilingüe, fiscal, fiscomisional, municipal, particular, rural, urbana, vespertina, nocturna, entre otras.

Este currículo partió de un proceso previo, primero se incrementó la carga horaria de Educación Física de dos horas a cinco horas pedagógicas a la semana para el nivel de Educación General Básica es decir desde $1^{\circ}$ año a $10^{\circ}$ año; al mismo tiempo se puso aplicó un programa que se denominó Aprendiendo en Movimiento destinándola tres horas y las dos horas restantes para la aplicación del currículo; con estos dos elementos se realizó un ajuste que fusionó este programa con el currículo, dando como resultado el currículo nacional vigente 2016.

El modelo educativo que mejor respondía a la expresión de la sociedad y al currículo fue el Constructivista Social (Posso, 2018a), este modelo se alinea a las necesidades de aprendizaje de las generaciones presentes y futuras, asegurando por medio de la praxis niveles altos en la calidad educativa y la autonomía investigativa y de adquisición de conocimientos, para desarrollarse como entes creativos, proactivos y emprendedores en una sociedad cada vez cambiante. 
La estructura del currículo se plantea desde el perfil del bachiller ecuatoriano, en base a la necesidad de la sociedad, planteando tres perfiles en las cuales los futuros ciudadanos tengan la competencia en Justicia, solidaridad e innovación; en este sentido los bachilleres serán Justos al comprender las necesidades de la sociedad e involucrarse directamente para aportar desde sus potencialidades y con ética, integridad, honestidad, respeto, responsabilidad, siendo parte del cambio y la transformación positiva; los bachilleres serán Innovadores al ser capaces de resolver diferentes problemáticas de la sociedad, a través del diálogo cooperativo y científico, demostrando creatividad, aplicando diferentes tipos de pensamiento y siendo autónomos de sus aprendizajes adquiridos; y por último los bachilleres serán Solidarios cuando demuestren responsabilidad social a través del trabajo con tolerancia, empatía e interacciones positivas, respetando la identidad y realidad nacional, la multiculturalidad y la multietnia de los pueblos y nacionalidades.

Una vez establecido el perfil de salida del bachiller ecuatoriano que responde a las preguntas ¿por qué enseñar?, ¿qué persona se desea formar? y ¿qué persona necesita la sociedad?; se plantean los objetivos generales del área de Educación Física que son una secuencia para alcanzar los perfiles de salida y responden a la pregunta ¿para qué enseñar Educación Física?, de estos se plantean lo objetivos por subnivel educativo del área de Educación Física que son una secuencia de logros por edades para alcanzar el perfil de salida y responden a la pregunta ¿para qué enseñar educación Física por subniveles educativos?

El currículo de Educación Física se desarrolla en dos niveles educativos el primero es Educación General Básica que a su vez tiene cuatro subniveles, Subnivel Preparatorio que lo conforma primero año y sus estudiantes oscilan entre las edades de 5 a 6 años; el Subnivel Elemental que lo conforman segundo, tercero y cuarto año y sus estudiantes oscilan entre 6 a 9 años.

El Subnivel Medio que lo conforman quinto, sexto y séptimo año y las edades de los estudiantes oscilan entre 9 y 12 años; el Subnivel Superior que lo conforman octavo, noveno y décimo año, sus estudiantes oscilan entre las edades de 12 a 15 años; y el Nivel Bachillerato que lo conforman primero, segundo y tercer año y sus estudiantes oscilan entre 15 a 19 años; esta clasificación lo adoptó para poder trabajar de forma flexible las destrezas con criterio de desempeño, en función a la libertad curricular y su contextualización. 
Los bloques curriculares son esquemas que agrupan destrezas con criterio de desempeño de iguales características y estos tienen progresión y continuidad de acuerdo con los niveles y subniveles educativos, en Educación Física son seis, cuatro principales o también llamados prácticas corporales y dos transversales; Posso (2018b) describe el primer bloque curricular:

1. Prácticas Lúdicas, las que plantean destrezas con criterio de desempeño que permitan crear y desarrollar en todas las clases actividades, juegos, dinámicas, entre otras;

2. Prácticas Gimnásticas plantean destrezas con criterio de desempeño que permiten crear y desarrollar en las clases actividades gimnásticas y coreográficas que mejoren la condición física;

3. Prácticas Corporales Expresivo Comunicativas plantean destrezas con criterio de desempeño que permitan crear y desarrollar cualquier actividad corporal que exprese y comunique, como el teatro, danza, mimo, payasearía, prácticas circenses, entre otras;

4. Prácticas Deportivas, esta tiene una característica la cual es que no se trabaja en los subniveles preparatorio y elemental, debido a que la técnica y táctica de los deportes se deben trabajar a una edad en la cual tengan la capacidad biológica para realizarlo, aquí se plantean destrezas con criterio de desempeño que permitan crear y desarrollar técnicas, estrategias y tácticas de diferentes deportes;

5. Construcción de la Identidad Corporal el cual es transversal y plantean destrezas con criterio de desempeño que permitan crear en conjunto con las destrezas con criterio de desempeño de los cuatro bloques de prácticas corporales la identidad individual y colectiva a través de la superación del movimiento; y, el

6. Relación de las Prácticas corporales y la salud, plantean destrezas con criterio de desempeño relacionados a la forma adecuada y saludable de realizar los ejercicios propuestos en las destrezas con criterio de desempeño de los cuatro bloques curriculares prácticas corporales.

Los contenidos curriculares conocidos en este currículo como destrezas con criterio de desempeño, se diseñó para que las clases sean desarrolladas bajo tres enfoques como lo menciona Posso, Barba, Castro, Nuñez y Marcillo (2019) el enfoque lúdico en que todas las actividades construidas por los estudiantes sean divertidas y placenteras; el enfoque inclusivo 
que todos los estudiantes participen activamente de la actividad desde lo motriz, cognitivo, social y afectivo; y, el enfoque de la corporeidad en que todos los estudiantes sean conscientes percibiendo su cuerpo a través del movimiento para la mejora de las actividades en todas las dimensiones; descartando por completo los enfoques tradicionales como eran el deportivista, el militarizado y el de capacidades físicas.

Estas destrezas con criterio de desempeño tienen una característica especial que a más de estructurarse con el contenido curricular que es abierto, existen tres elementos más, la destreza que no es necesariamente motriz sino también cognitiva, y que es el generador de todas las actividades propuestas en ellas; el nivel de complejidad es la dificultad que se le plantea a la realización del contenido curricular; y, el contexto que no necesariamente se puede ver a simple vista sino que en muchas ocasiones está implícita.

Las 181 destrezas con criterio de desempeño que se distribuyen en los seis bloques curriculares y en los niveles y subniveles del currículo de Educación Física, desarrollan de manera completa los aprendizajes básicos tanto imprescindibles que son los que se deben cumplir para garantizar la estandarización de conocimientos y los aprendizajes básicos deseables que son los que al cumplirse busca la excelencia y la calidad educativa y al mismo tiempo cumplir con el perfil de salida del bachiller ecuatoriano.

Las destrezas con criterio de desempeño se aplican con orientación a las adaptaciones curriculares, en cumplimiento al desarrollo del enfoque inclusivo; como se lo planteó anteriormente todos los estudiantes participan activamente de las actividades, es decir todos los estudiantes con necesidades educativas especiales asociados a la discapacidad física, intelectual, sensorial y psico social y los no asociados a la discapacidad deben participar de las clases,

No obstante, desde el punto constructivista cooperativo, si no puede hacer trabajo motriz por su discapacidad, realizará el trabajo intelectual como selección de alternativas, mejores soluciones, selección, análisis, síntesis, entre otras; sino realizará trabajo direccionado a lo social como comunicación efectiva, distribución y asignación de tareas, relaciones interpersonales, ente otros; y por último realizar trabajo orientado a la afectividad, como motivación, valores, principios, voluntad y recreativo como lo plantean Posso, Aponte, Zapata y Betancourt (2020) al mencionar que en el trabajo inclusivo se aplica el recurso de la recreación para alcanzar los objetivos deseados. 
Estas adaptaciones curriculares permiten que los estudiantes participen de la actividad desde sus potencialidades, rompiendo el paradigma de una Educación Física únicamente motriz y castigadora, sino de una Educación Física participativa e incluyente; tomando en cuenta este apartado Ullaguari (2016) menciona "La educación inclusiva garantiza los principios de igualdad, la accesibilidad, reconociendo y respetando las potencialidades y capacidades individuales, necesita un entorno inclusivo, una sociedad inclusiva y de derecho"

Como se ha mencionado anteriormente este currículo se ha caracterizado por ser diferente a los demás currículos ecuatorianos está construido desde la idea de ser flexible, abierto y participativo en aprovechamiento de la libertad curricular de los docentes. Este es flexible debido a que las destrezas con criterio de desempeño interpretadas desde la comprensión de los cuatro elementos que les componen se contextualizan de acuerdo al propósito del aprendizaje, Zabala (2012) aporta al decir que la contextualización curricular es "un proceso a través del cual, las propuestas curriculares se ajustan a los parámetros particulares de los diversos entornos, instituciones y colectivos donde se aplicarán”,

Lo anterior señalado esto quiere decir teniendo una óptica de las necesidades y características de los estudiantes frente a las destrezas con criterio de desempeño, las experiencias previas de los estudiantes, los aprendizajes obtenidos, la realidad institucional y poblacional, la infraestructura y los recursos, el conocimiento del docente, entre otras; todo esto permite seleccionar, modificar, contextualizar, desagregar y descartar las 181 destrezas con criterio de desempeño del currículo nacional de Educación Física.

Es abierta porque permite la gradación de las 181 destrezas con criterio de desempeño, es decir que los docentes elijen los contenidos a tratar y su nivel de complejidad, contextualizándolas a los intereses y necesidades que tengan los estudiantes, a la realidad institucional y de la comunidad, sin dejar de lado las capacidades y el ritmo de aprendizaje de los estudiantes.

Es participativa porque toda la comunidad educativa se involucra en el proceso de enseñanza y aprendizaje, al poder sugerir y aportar información y actividades, proponer los ritmos de aprendizajes, contextualizar los conocimientos, expresar sus dudas e inquietudes, entre otras; es decir en este currículo es necesario que exista participación de la comunidad educativa, aportando con sus saberes culturales para poder contextualizarlos en las destrezas con criterio 
de desempeño, con la carga de valores y principios desde la familia para ser reforzados transversalmente en cada actividad diseñada.

Cada subnivel tiene destrezas con criterio de desempeño de cinco y seis bloques curriculares, las cuales deben gradarse y desagregarse según el propósito de aprendizaje que el profesor estime para cada año de Educación General Básica y Bachillerato.

Tabla 1

Matriz de destrezas con criterio de desempeño por bloque y nivel

\begin{tabular}{|c|c|c|c|c|c|}
\hline $\begin{array}{l}\text { Nivel } \\
\text { Bloque }\end{array}$ & $\begin{array}{c}\text { Preparatoria } \\
1^{\circ}\end{array}$ & $\begin{array}{l}\text { Elemental } \\
2^{\circ}, 3^{\circ} \text { y } 4^{\circ}\end{array}$ & $\underset{6^{\circ} \text { y } 7^{\circ}}{5^{\circ},}$ & $\begin{array}{l}\text { Superior } \\
\quad 9^{\circ} \text { y } 10^{\circ}\end{array}$ & $\begin{array}{c}8^{\circ}, \quad \text { Bachillerato } \\
1^{\circ}, 2^{\circ} \text { y } 3\end{array}$ \\
\hline $\begin{array}{l}\text { Prácticas } \\
\text { Lúdicas }\end{array}$ & 6 & 10 & 10 & 9 & 5 \\
\hline $\begin{array}{l}\text { Prácticas } \\
\text { Gimnásticas }\end{array}$ & 6 & 7 & 7 & 5 & 5 \\
\hline $\begin{array}{l}\text { Prácticas } \\
\text { corporales } \\
\text { expresivo } \\
\text { comunicativas }\end{array}$ & 7 & 9 & 6 & 8 & 6 \\
\hline $\begin{array}{l}\text { Prácticas } \\
\text { Deportivas }\end{array}$ & ----- & ----- & 7 & 7 & 9 \\
\hline $\begin{array}{l}\text { Construcción } \\
\text { de la Identidad } \\
\text { corporal }\end{array}$ & 4 & 7 & 5 & 4 & 6 \\
\hline $\begin{array}{l}\text { Relación entre } \\
\text { las prácticas } \\
\text { corporales y la } \\
\text { salud }\end{array}$ & 2 & 6 & 5 & 6 & 7 \\
\hline $\begin{array}{l}\text { Total } \\
181 \text { DCD }\end{array}$ & 25 & 39 & 40 & 39 & 38 \\
\hline
\end{tabular}


La desagregación y la gradación de las destrezas con criterio de desempeño nacen del hecho que pertenecen a todo un subnivel, es decir una destreza con criterio de desempeño debe desarrollarse en los tres años que conforman un subnivel el cual se puede ejemplificar de la siguiente forma: el subnivel medio tiene una sola destreza para $5^{\circ}, 6^{\circ}$ y $7^{\circ}$ año, en este sentido el docente deberá construir según su criterio apoyándose de la contextualización, el propósito del aprendizaje y la edad de los estudiantes, que parte desarrolla en $5^{\circ}$, que parte desarrolla en $6^{\circ}$ y que parte desarrolla en $7^{\circ}$, al decir que parte desarrolla en los tres años, el docente aumentará contenido curricular específico para cada año, esa acción se le llama gradar y qué parte debe quitar para cada uno de los tres años esa acción se le llama desagregar.

De la misma forma estas destrezas con criterio de desempeño se deben desarrollar en las clases de Educación Física no aisladas, sino en conjunto como una unidad, es decir el docente deberá trabajar más de tres destrezas con criterio de desempeño de tres diferentes bloques curriculares en una actividad, y al final de una unidad didáctica que pertenece al tercer nivel de concreción se terminarán de desarrollar por completo las destrezas abordadas en las clases.

El currículo también tiene criterios de evaluación que responde a la pregunta ¿cómo evaluar? las cuales se estructuran en matrices, agrupando destrezas con criterio de desempeño destrezas estas responden a la pregunta ¿qué enseñar? Cada una de ellas tienen un indicador de evaluación y esta responde a la pregunta ¿qué evaluar? Entonces el ¿qué enseñar? y el ¿qué evaluar? son la naturaleza misma del proceso de enseñanza aprendizaje, por lo que la acción a seguir es desagregar el indicador de evaluación para que se alinee a la destreza con criterio de desempeño; cabe indicar que esta acción de acuerdo con el ajuste curricular permitirá cumplir con los objetivos del área de cada subnivel y alinearse a los perfiles de salida.

Con lo anteriormente mencionado se cumple con un currículo abierto y flexible, dependiendo solamente del docente la responsabilidad interpretar y de definir un contenido que no está señalado en el currículo, la posibilidad de gradar y desagregar las destrezas con criterio de desempeño, la contextualización del contenido curricular y el nivel de dificultad, el involucramiento de todos los docentes del área de Educación Física, la participación de todos los docentes del segundo nivel de concreción, del apoyo de toda la comunidad educativa.

Desde este panorama el currículo es futurista, si existe la intención de ver su proyección constructivista versus la interpretación desde una mirada conductista. Este aporte afecta a toda la comunidad educativa estudiantes, profesores, autoridades y familiares, que no están 
capacitados o no pueden interpretar las 181 destrezas con criterio de desempeño, al desglosarles en sus cuatro elementos, ponerlos el contenido curricular para cada año y quitar lo que no se va a desarrollar grado por grado.

Después de haber realizado la reseña al Currículo Nacional del Ecuador de Educación Física, se puede dar cuenta que los principios filosóficos, pedagógicos, sociales, etnográficos, psicológicos no están enmarcadas al planteamiento de cada subnivel. También hay que señalar que las destrezas con criterio de desempeño no están bien construidas, no están en secuencia lógica, no siempre reflejan un orden progresivo y no están contextualizada a la realidad del país.

También se puede mencionar que no está clara el criterio de evaluación por cada subnivel y por lo tanto se confunde con los indicadores de evaluación, se podría conocer qué se va a evaluar desglosando estos indicadores de evaluación en varios indicadores de logro para que el cierre del proceso enseñanza aprendizaje constituya el reflejo de las necesidades sobre la evaluación por procesos.

Todo lleva a pensar que si los docentes de Educación Física cumplen con la aplicación del currículo en todas sus expresiones interpretadas a la contextualización, se podría alcanzar en conjunto dos cosas el primero el perfil del bachiller ecuatoriano y segundo la autonomía de la actividad física y el ejercicio físico, para que los estudiantes vuelvan a realizar acciones motrices sin ningún direccionamiento y en cualquier momento de la vida.

\section{REFERENCIAS}

Posso, R. (2018a). Guía de estrategias metodológicas para Educación Física. Quito: Ministerio de Educación. Recuperado https://educacion.gob.ec/wpcontent/uploads/downloads/2019/01/GUIA-METODOLOGICA-EF.pdf

Posso, R. (2018b). Propuesta de Estrategias Metodológicas aplicadas a la Educación Física (Tesis de maestría). Universidad Internacional de la Rioja, Logroño. Recuperado de https://reunir.unir.net/handle/123456789/7439

Posso, R., Barba, L., Castro, R., Nuñez, F. y Marcillo, J. (2019). Enfoque lúdico como estrategia en el contexto de la Educación Física ecuatoriana: una revisión sistemática. Lecturas: Educación Física y Deportes, 24(258), 86-105 Recuperado de https://www.efdeportes.com/index.php/EFDeportes/article/view/1531/1021 
Posso, R., Aponte, J., Zapata, M. y Betancourt, A. (2020). Aproximación fenomenológica y hermenéutica de los expertos en recreación, sobre las teorías del ocio y el tiempo libre en el proceso de socialización en las instituciones educativas, Revista Científica Olimpia, $\quad$ 17, 78-91. Recuperado de https://revistas.udg.co.cu/index.php/olimpia/article/view/1269/2293

Ullaguari, S. (2016). La Educación Física ecuatoriana con enfoque inclusivo en la diversidad. Lecturas: Educación Física y Deportes, (217), 1-15 Recuperado de https://www.efdeportes.com/efd217/la-educacion-fisica-con-enfoque-inclusivo.htm

Zabalza, M. (2012). Territorio, cultura y contextualización curricular. Interacções, 22, 6-33. Recuperado de https://revistas.rcaap.pt/interaccoes/article/view/1534/1225 